\title{
Recruitment of Calcium-Permeable AMPA Receptors during Synaptic Potentiation Is Regulated by CaM-Kinase I
}

\author{
Eric S. Guire, Michael C. Oh, Thomas R. Soderling, and Victor A. Derkach \\ Vollum Institute, Oregon Health \& Sciences University, Portland, Oregon 97225
}

\begin{abstract}
$\mathrm{Ca}^{2+}$-permeable AMPA receptors (CP-AMPARs) at central glutamatergic synapses are of special interest because of their unique biophysical and signaling properties that contribute to synaptic plasticity and their roles in multiple neuropathologies. However, intracellular signaling pathways that recruit synaptic CP-AMPARs are unknown, and involvement of CP-AMPARs in hippocampal region CA1 synaptic plasticity is controversial. Here, we report that intracellular infusion of active CaM-kinase I (CaMKI) into cultured hippocampal neurons enhances miniature EPSC amplitude because of recruitment of CP-AMPARs, likely from an extrasynaptic pool. The ability of CaMKI, which regulates the actin cytoskeleton, to recruit synaptic CP-AMPARs was blocked by inhibiting actin polymerization with latrunculin A. CaMK regulation of CP-AMPARs was also confirmed in hippocampal slices. CA1 long-term potentiation (LTP) after theta bursts, but not high-frequency tetani, produced a rapid, transient expression of synaptic CP-AMPARs that facilitated LTP. This component of TBS LTP was blocked by inhibition of CaM-kinase kinase (CaMKK), the upstream activator of CaMKI. Our calculations show that adding CP-AMPARs numbering $<5 \%$ of existing synaptic AMPARs is sufficient to account for the potentiation observed in LTP. Thus, synaptic expression of CP-AMPARs is a very efficient mechanism for rapid enhancement of synaptic strength that depends on CaMKK/ CaMKI signaling, actin dynamics, and the pattern of synaptic activity used to induce CA1 LTP.
\end{abstract}

Key words: AMPA receptor; CaM-kinase; LTP; calcium; hippocampus; synaptic plasticity

\section{Introduction}

In the mammalian forebrain, AMPA-type glutamate receptors (AMPARs), which are tetramers of glutamate receptor 1 (GluR1)-GluR4 subunits, mediate rapid excitatory synaptic transmission. The presence of the edited GluR2 subunit critically determines AMPAR properties (for review, see Isaac et al., 2007). AMPARs lacking the edited GluR2 subunit are calciumpermeable (CP-AMPARs), exhibit rapid kinetics and high conductance, and are inwardly rectifying. Synaptic localization of CP-AMPARs can be modulated by neuronal activity, neuronal insults (e.g., ischemia, excitotoxicity), antipsychotics, and drugs of abuse, and are implicated in several neuropathologies (e.g., Alzheimer's) (for review, see Kwak and Weiss, 2006; Isaac et al., 2007; Liu and Zukin, 2007).

The role of CP-AMPARs in CA1 long-term potentiation (LTP), a model of associative learning and memory, is currently controversial. LTP can be induced using multiple paradigms including high-frequency tetanic stimulation (HFS), pairing of presynaptic and postsynaptic depolarizations, and theta-burst stimulation (TBS). In the mature hippocampus, CA1 pyramidal cell AMPA receptors are composed predominantly of GluR1 and GluR2 (Monyer et al., 1991; Craig et al., 1993; Geiger et al., 1995;

Received Jan. 28, 2008; revised April 9, 2008; accepted May 5, 2008.

This work was supported by National Institutes of Health Grant R01 NS27037. We thank Sean Nygaard for excellent preparation of cultured hippocampal neurons and other technical assistance.

Correspondence should be addressed to Thomas R. Soderling, Vollum Institute L-474, Oregon Health \& Sciences University, 3181 Southwest Sam Jackson Park Road, Portland, OR 97225. E-mail: soderlit@ohsu.edu. DOI:10.1523/JNEUROSCI.0384-08.2008

Copyright $\odot 2008$ Society for Neuroscience $\quad$ 0270-6474/08/286000-10\$15.00/0
Wenthold et al., 1996; Sans et al., 2003). Application of CPAMPAR inhibitors has little or no effect on basal CA1 synaptic transmission (Ju et al., 2004; Plant et al., 2006; Sutton et al., 2006; Adesnik and Nicoll, 2007), indicating a lack of synaptic CPAMPARs. One report observed that pairing-induced LTP gave a transient incorporation of synaptic CP-AMPARs (Plant et al., 2006), whereas two other reports did not see this subunit recomposition using either pairing-induced LTP or HFS-LTP (Adesnik and Nicoll, 2007; Gray et al., 2007). A fourth report indicates that the ability of HFS to induce AMPAR subunit recomposition may be dependent on the age of the rats (Y. Lu et al., 2007).

Expression of LTP uses multiple signaling cascades, some of which may be age or protocol dependent (Selcher et al., 2003; Yasuda et al., 2003; Schmitt et al., 2005; Li et al., 2006). Thus, identification of pathways that regulate CP-AMPARs may explain their variable participation in LTP, will be essential for understanding their physiological roles, and may have implications for neuropathologies. To investigate synaptic recruitment of CPAMPARs, we combined whole-cell recordings from cultured hippocampal neurons with field recordings from acute slices. For LTP, we used a TBS induction protocol because it mimics the endogenous hippocampal firing patterns during active exploration and learning (Feder and Ranck, 1973; Otto et al., 1991), and field recordings of synaptic strength to maintain the physical integrity of the neurons and, therefore, endogenous signaling pathways. We focused on CaM-kinase I (CaMKI) and its upstream activator CaM-kinase kinase (CaMKK), which are critical components of TBS-LTP through cross talk with Ras-extracellular signal-regulated protein kinase (Ras-ERK) signaling (Schmitt et al., 2005). The Ras-ERK pathway contributes to TBS-LTP 
(Selcher et al., 2003) and regulates activity-dependent synaptic trafficking of GluR1 (Zhu et al., 2002) whose C terminus appears to be essential for structural plasticity of synaptic spines (Kopec et al., 2007).

\section{Materials and Methods}

Hippocampal culture and recordings. Rats were housed in the Oregon Health \& Science University Animal Care Facility and all procedures were approved by the Institutional Animal Care and Use Committee. Voltage-clamp recordings of spontaneous miniature EPSCs (mEPSCs) were performed at $-70 \mathrm{mV}$ on pyramidal cells from $14-21 \mathrm{~d}$ in vitro (DIV) rat hippocampal cultures at room temperature. For measurements of average channel conductance of synaptic AMPARs, we used nonstationary fluctuation analysis of mEPSCs (see below). To assess the role of CaMKI in synaptic plasticity, constitutively active CaMKI (caCaMKI) (Wayman et al., 2004) or heat-inactivated control (inactCaMKI) was infused through the patch pipette, with or without latrunculin A.

Hippocampal cultures were prepared essentially as described previously (Oh and Derkach, 2005). Cell seeding density was 20,000-25,000 cells $/ \mathrm{cm}^{2}$. To isolate spontaneous mEPSCs, the extracellular solution contained (in mM) $125 \mathrm{NaCl}, 2.5 \mathrm{KCl}, 1 \mathrm{MgCl}_{2}, 2 \mathrm{CaCl}_{2}, 25$ HEPES, 33 D-glucose, 0.025 D-APV, 0.003 strychnine, 0.05 picrotoxin or 0.01 gabazine, and 0.0005 TTX. Patch pipettes for whole-cell recordings were filled with (in mM) 100 Cs-methanosulfonate, $25 \mathrm{CsCl}, 2 \mathrm{MgCl}_{2}, 10$ HEPES, 0.4 EGTA, 4 ATP, 0.4 GTP, 10 phosphocreatine, 0.1 spermine, and 0.05-0.1 Alexa 594, pH 7.3. Glutathione $S$-transferase-tagged recombinant caCaMKI (Wayman et al., 2004) was expressed in Escherichia coli, purified on a glutathione-sepharose column, and added to the patch-pipette solution from a concentrated stock solution. Heat-inactivated CaMKI was produced by heating an aliquot of stock caCaMKI at $95^{\circ} \mathrm{C}$ for $15 \mathrm{~min}$; loss of activity was verified by kinase assay (see Fig. 2 F). Stock solution (1 $\mathrm{mM}$ ) of latrunculin A (Sigma) was prepared in ethanol. Currents were recorded at a $2 \mathrm{kHz}$ frequency bandwidth. Individual mEPSCs were detected using the template algorithm (AxoGraph 4.0 or pCLAM9; Molecular Devices), and their representative amplitudes and kinetic properties were determined based on the averaged mEPSC (100-300 currents averaged) or from corresponding cumulative distributions. These measurements of spontaneous activity were made every $5 \mathrm{~min}$. AMPA currents were evoked by $10-15 \mathrm{~ms}$ pressure puffs from a patch-pipette filled with 0.3-0.5 mm AMPA, 0.1 mm cyclothiazide, and 0.1 mu Alexa 594. The tip of the patch pipette was positioned 100-150 $\mu \mathrm{M}$ away from the soma on second-order apical dendrites of the pyramidal neuron for a local activation of dendritic AMPARs. A local suction pipette was also positioned across and in a close proximity to the application pipette to ensure a confined AMPA application. The precision of application was monitored through Alexa 594 fluorescence. Pyramidal neurons were identified by a prominent presence of dendritic spines visualized by fluorescence of intracellular Alexa 594. To further confirm their identity, in several experiments we stained recorded cells with $\alpha$-GAD65/67 antibody, revealing they are not GAD65/67 positive and, thus, are not interneurons.

Brain slice physiology. Young adult rats (4-6 weeks old) were anesthetized with pentobarbital $(60 \mathrm{mg} / \mathrm{kg}$, i.p.) and decapitated. CA1 stratum radiatum field EPSPs (fEPSPs) (1.2-1.4 mV basally, $\sim 25 \%$ maximally) were recorded by Schaffer collateral (SC) stimulation of submerged hippocampal slices (transverse, $400 \mu \mathrm{M}, 30-32^{\circ} \mathrm{C}$ ). Long-term potentiation was induced using three trains (20 s intertrain interval) of HFS $(100 \mathrm{~Hz}$, $1 \mathrm{~s})$ or TBS: four pulses per burst $(100 \mathrm{~Hz})$, five bursts per train $(5 \mathrm{~Hz})$.

Submerged hippocampal slices were suspended in pairs on a nylon mesh in a small $(\sim 350 \mu \mathrm{l})$ recording chamber and perfused with artificial CSF (ACSF) at a rate of $2.5-3 \mathrm{ml} / \mathrm{min}$, allowing rapid drug perfusion of the slices (dead volume of perfusion tubing also $\sim 350 \mu \mathrm{l}$ ) as confirmed in Figure $5 \mathrm{~A}$ (APV treatment). For rapid application of 1-trimethylammonio-5-(1-adamantane-methylammoniopentane)dibromide [IEM-1460 (IEM); $30 \mu \mathrm{M}$, prepared from a $100 \mathrm{~mm}$ aqueous stock solution; Tocris Bioscience], the flow of solution coming into the chamber was switched from ACSF to ACSF plus IEM immediately after the last train of HFS or TBS. For 1,8-naphthoylene benzimidazole-3-carboxylic acid (STO-609)-treated slices (Tocris Bioscience; $5 \mu \mathrm{M}$, prepared from a $25 \mathrm{~mm}$ stock solution in $0.1 \mathrm{~N} \mathrm{NaOH}$ ), treatment began in the recording chamber 30 min before LTP induction and STO washout began immediately after the last train of HFS or TBS. Experiments were otherwise performed as described (Schmitt et al., 2005), except as noted. Test-pulse stimulation was administered once every $20 \mathrm{~s}$. One minute fEPSP averages are shown. NMDAR fEPSPs were pharmacologically isolated at basal test-pulse intensity by blocking AMPA/kainate receptors with 10 $\mu \mathrm{M}$ CNQX (Tocris Bioscience) in the presence of low $\mathrm{Mg}^{2+}(0.1 \mathrm{~mm})$. The resulting responses were confirmed to be NMDAR field potentials by blockade with $50 \mu \mathrm{M}$ APV (Tocris Bioscience).

In vitro phosphorylation assay. Phosphorylation assays were performed essentially as described using syntide-2 (Tokumitsu et al., 1995; Barria et al., 1997a). The same caCaMKI (Wayman et al., 2004) was used as in the infusion experiments described above.

Nonstationary fluctuation analysis. We assumed that the majority of recorded mEPSCs were monoquantal (Bergles et al., 1999). This was also confirmed by the absence of correlation between their amplitude and decay kinetics (data not shown). Because of their monoquantal character, the fluctuations in their waveform should represent the stochastic behavior of synaptic AMPARs, as apposed to fluctuations of evoked EPSCs which are additionally determined by variable quantum number and asynchronization of quantal release. Therefore, we applied nonscaled nonstationary fluctuation analysis (Sigworth, 1980; Derkach et al., 1999; Oh and Derkach, 2005) instead of scaling, which would be preferable for evoked EPSCs (Traynelis et al., 1993; Benke et al., 1998). For each measurement, 100-200 mEPSCs were carefully selected based on the following criteria: stable baseline $10 \mathrm{~ms}$ before and $10 \mathrm{~ms}$ after mEPSC, fast rise time $(<0.5 \mathrm{~ms})$ allowing a precise alignment, and no overlapping $\mathrm{mEP}$ SCs or spurious fluctuations during the decay phase $(25 \mathrm{~ms})$. mEPSCs were aligned by their point of maximal rise, averaged, and their mean value and variance was calculated (see Fig. $2 A, B$, insets). In all cells tested, the variance was essentially a linear function of mean current (see Fig. $2 A, B$ ), indicating a low open probability of synaptic AMPARs, consistent with their low occupancy by released glutamate (Bergles et al., 1999; Mainen et al., 1999). We therefore fitted the relationship with the following equation: $\operatorname{var}=i I+$ varb, where $i$ was the mean single-channel AMPA current, $I$ the mean current, and varb the variance of background noise. Channel conductance was then calculated as $\gamma=i / V$, where $V$ was the holding membrane potential (assuming $0 \mathrm{mV}$ for reversal potential). There was no correlation between conductance and average mEPSC amplitude, mean decay time or background noise. Cells were discarded if access resistance varied during measurements by $>15 \%$. We calculated channel conductance under conditions of break in (initial 2-5 min and used as "same cell control"), 15 min of CaMKI infusion $(n=8)$, and an additional 15 min of IEM ( $30 \mu \mathrm{M} ; n=4)$ bath application (see Fig. $2 C$ ).

Cable filtration can be a factor for underestimation of single-channel conductance (Benke et al., 2001). However, other factors can in contrast lead to overestimation of it during nonstationary fluctuation analysis of evoked EPSCs. In particular, asynchronization in transmitter release in both single synapses and between synapses is an additional contributor to estimated variance (Traynelis et al., 1993) and may result in significantly higher values for $\gamma$ (V. A. Derkach, unpublished observation). The scaling procedure cannot compensate for that, but the analysis of mEPSCs should produce more correct and lower values. This notion is consistent with observations of others that $\gamma$ estimates for synaptic AMPARs were several-fold higher if based on EPSCs versus mEPSCs (Mameli et al., 2007). Our estimates of $\gamma$ for synaptic AMPARs at basal conditions are also in accord with the recent work of Cull-Candy and Gebhardt (2006) (their Fig. 2) on endogenous AMPARs in CA1 neurons. At a low receptor occupancy by agonist, the case for synaptically released glutamate, the main conductance state for AMPARs in CA1 pyramidal neurons was 3.9 pS. Thus, we felt that the low picosiemen values for $\gamma$ that we and others obtained for endogenous AMPARs under basal conditions were well grounded and were used in our modeling. 


\section{Results}

CaM-kinase I activity promotes synaptic incorporation of CP-AMPARs

The CaMKK/CaMKI cascade is involved in multiple aspects of activity-dependent neuronal development and plasticity (Wayman et al., 2004, 2006; Saneyoshi et al., 2008), including TBS-LTP (Schmitt et al., 2005), in part because of regulation of actin dynamics (Wayman et al., 2004, 2006; Saneyoshi et al., 2008) and cross talk with the Ras/MEK (mitogen activated protein kinase kinase)/ERK pathway (Schmitt et al., 2004). Because actin dynamics and ERK signaling are both thought to regulate activitydependent trafficking of AMPARs to synapses (Matsuzaki et al., 2004; Korkotian and Segal, 2007), we investigated whether CaMKK/CaMKI might regulate expression of synaptic AMPARs. We focused on CP-AMPARs because of their potential role in the initial phase of CA1 LTP (Plant et al., 2006) (but see Adesnik and Nicoll, 2007; Gray et al., 2007), their ability to be uniquely identified, and their potential for subsequent downstream signaling via $\mathrm{Ca}^{2+}$ influx.

Infusion of activated CaMKI into cultured hippocampal pyramidal cells (14-20 DIV) produced a significant potentiation of AMPAR mEPSC amplitude that plateaued within 15 min (Fig. $1 A, B)$. This potentiation required CaMKI activity, because infusion of inactCaMKI had no effect (Fig. $1 B, C$ ). We did not observe any significant effects of CaMKI or inactCaMKI infusion of mEPSC frequency (data not shown). To assess the potential involvement of CP-AMPARs in mEPSC potentiation, we used IEM-1460, a well characterized rapid and reversible inhibitor, which shows greater selectivity than philanthotoxin-433 for CPAMPARs versus GluR2-containing AMPARs and NMDARs (Magazanik et al., 1997; Buldakova et al., 1999, 2007; Samoilova et al., 1999; Gray et al., 2007). For example, IEM has no effect on basal AMPA EPSCs from CA1 pyramidal neurons, which do not contain CP-AMPARs (Monyer et al., 1991; Craig et al., 1993; Geiger et al., 1995; Wenthold et al., 1996; Sans et al., 2003), whereas IEM partially suppresses AMPA EPSCs of CA1 interneurons (Buldakova et al., 2007), which do contain some CPAMPARs (Racca et al., 1996; Laezza and Dingledine, 2004). The best evidence of IEM specificity for synaptic GluR2-lacking AMPARs comes from GluR2 knock-out mice. Basal transmission at CA1 synapses was efficiently inhibited in that mouse, but not in the wild type (Gray et al., 2007). In our experiments, IEM had no effect on mEPSC amplitude when heat-inactivated CaMKI was infused (Fig. $1 \mathrm{~B}$ ), indicating that CP-AMPARs do not contribute to basal synaptic transmission in these cultured neurons. In contrast, the CaMKI-mediated potentiation of mEPSC amplitude was completely reversed by IEM (Fig. $1 A, B)$. Because CPAMPARs exhibit more rapid kinetics (Grosskreutz et al., 2003; Oh and Derkach, 2005; Isaac et al., 2007), we also examined the decay kinetics of mEPSCs, which are controlled by deactivation of synaptic AMPARs, as additional confirmation of synaptic CPAMPAR recruitment. Infusion of active CaMKI significantly shortened mEPSC decay time, an effect also reversed by IEM (Fig. $1 C)$. As with mEPSC amplitude, inactive CaMKI with or without IEM had no effect on mEPSC decay kinetics (Fig. 1C). Thus, both the IEM sensitivity of potentiation and the acceleration of mEPSC decay kinetics indicated that CaMKI activity initiated the appearance of CP-AMPARs at synapses.

\section{CaMKI enhances synaptic strength by increasing average} channel conductance of synaptic AMPARs

GluR2-lacking AMPARs have significantly higher channel conductance than GluR2-containing AMPARs (Swanson et al., 1997;
A
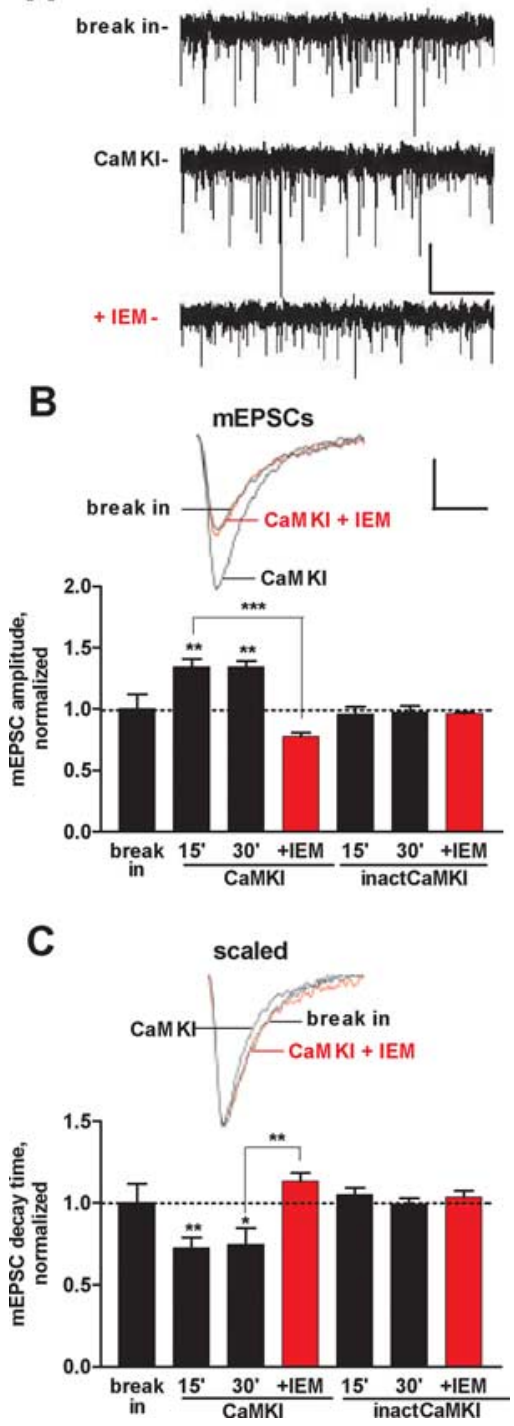

Figure 1. CaMKI activity promotes synaptic CP-AMPAR expression. $\boldsymbol{A}$, Representative traces of spontaneous AMPAR mEPSCs from a single experiment during the initial 5 min of infusion of activated CaMKI ( $500 \mathrm{~nm}$ ) (break in, top trace). After $15 \mathrm{~min}$ of CaMKI infusion, the amplitude of $\mathrm{mEPSC} s$ was significantly potentiated (middle trace). This potentiation was reversed by subsequent treatment for 15 min with a selective inhibitor of CP-AMPARs, IEM-1460 (30 $\mu \mathrm{m}$, bottom trace). Calibration: 10 pA, 2 s. $\boldsymbol{B}, \boldsymbol{C}$, Infusion of activated CaMKI ( $500 \mathrm{~nm}$ ) into cultured hippocampal pyramidal cells increased AMPAR mEPSC amplitude $(\boldsymbol{B})$ and decreased decay time (C, $90-$ $10 \%$ decay time) compared with break in $(n=7)$. In contrast, infusion of inactive CaMKI (heat inactivated; see Fig. $2 F)$ had no effect on the amplitude and decay time of mEPSCs $(n=4)$. Both effects of active CaMKI were reversed by bath application of IEM (30 $\mu \mathrm{m})$ beginning $15 \mathrm{~min}$ after break in $(n=4-5)$, whereas IEM had no significant effects on mEPSCs recorded from cells infused with inactive CaMKI $(n=3)$. Insets, Representative traces of mEPSCs showing responses during the initial 5 min (break in), after 15 min infusion of active CaMKl, and after an additional 15 min with IEM (red traces). Calibration: 5 pA, 5 ms. $^{*} p<0.05$; ${ }^{* *} p<0.01$; ${ }^{* * *} p<$ 0.001. Error bars indicate SEM.

Oh and Derkach, 2005). Furthermore, the channel conductance of GluR1 homomers can be significantly enhanced by CaMKII phosphorylation of Ser831 in the C terminus of the GluR1 subunit in contrast to GluR2-GluR1 heteromers (Derkach et al., 1999; Derkach, 2003). Therefore, one would predict that synaptic incorporation of CP-AMPARs by CaMKI should be accompanied by increased averaged channel conductance of synaptic AMPARs.

Under basal conditions, the channel conductance $(\gamma)$ of syn- 
aptic AMPARs, calculated by nonstationary fluctuation analyses (Fig. 2A), was variable from cell to cell but averaged $3.8 \pm 0.48 \mathrm{pS}(n=8)$ (Fig. $2 C)$. This value is consistent with the conductance of GluR1/GluR2 (edited) heteromers (3.1 \pm $0.3 \mathrm{pS})$, but not homomeric GluR1 $(12.2 \pm 1.3 \mathrm{pS})$ (Derkach et al., 1999; Oh and Derkach, 2005). CaMKI infusion significantly increased channel conductance to $4.9 \pm 0.50 \mathrm{pS}(n=8 ; p=0.013)$, and IEM reversed this increase to $3.5 \pm 0.46 \mathrm{pS}$ $(n=4 ; p=0.031)$ (Fig. $2 B, C)$. To further clarify these analyses, effects of CaMKI infusion and IEM treatment were normalized for each cell to the initial conductance after break in. These normalized changes showed a significant increase in conductance by CaMKI by $\sim 30 \%$ that was fully reversed by IEM (Fig. 2D). Importantly, changes in mEPSC amplitude strongly correlated with changes in channel conductance (Fig. 2E). This suggests that the major mechanism for potentiation of synaptic strength by CaMKI was regulation of average channel conductance, likely through synaptic incorporation of IEMsensitive CP-AMPARs.

Synaptic AMPARs can be potentiated by multiple mechanisms: modulation of receptor number through trafficking, alteration of subunit composition, and phosphorylation of the GluR1 C terminus (Derkach et al., 2007). For example, phosphorylation of Ser831 by CaMKII (Barria et al., 1997a) can increase single channel conductance of homomeric GluR1 AMPARs (Derkach et al., 1999; Oh and Derkach, 2005). Because there can be overlap in substrate phosphorylation by the CaMK family members, we examined whether CaMKI might also phosphorylate the C terminus of GluR1, which might account for the observed enhancement of channel conductance. Although CaMKI and CaMKII both phosphorylated the synthetic peptide syntide (Syn), only CaMKII phosphorylated the GluR1 C terminus of GluR1 (Fig. 2 F). Lack of in vitro phosphorylation of Ser831 by CaMKI is not surprising because the sequence surrounding Ser831 (PQQQS) does not conform to a consensus CaMK phosphorylation motif which contains an Arg in the P-3 position (RXXS/T). This result is also consistent with the finding that enhanced Ser831 phosphorylation during TBS-LTP is not suppressed by the CaMKK inhibitor STO-609 (Schmitt et al., 2005). Thus, based on four independent criteria (IEM-sensitivity, faster decay kinetics, increased channel conductance, and lack of phosphorylation of Ser831 by CaMKI) we conclude that CaMKI potentiates mEPSC amplitude through recruitment of $\mathrm{CP}$ AMPARs at synapses.

\section{Recruitment of synaptic CP-AMPARs by CaMKI requires actin polymerization}

We investigated the potential regulatory role of actin polymerization for several reasons. First, actin polymerization is essential for activity-dependent AMPAR trafficking, including $\mathrm{Ca}^{2+}$ -

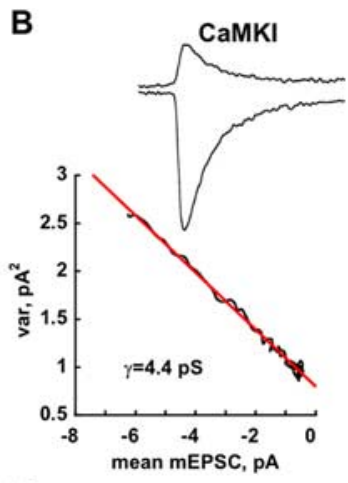

C
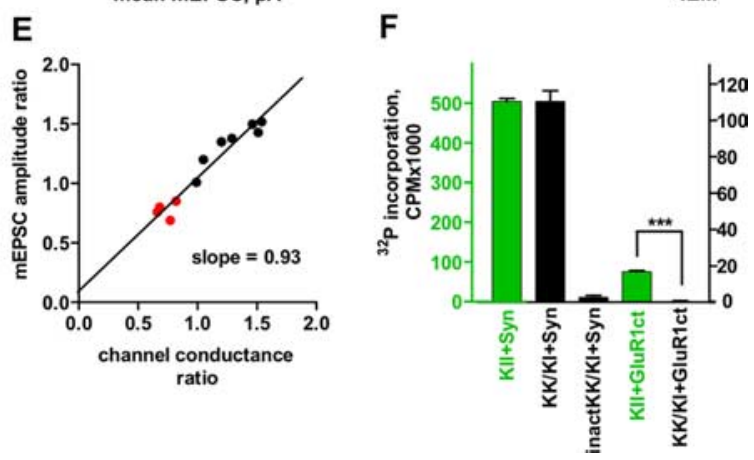

Figure 2. CaMKI increases channel conductance of synaptic AMPARs. $A, B$, Representative nonstationary fluctuation analyses for $\mathrm{mEPSC}$ recorded in the same cell after break in $(\boldsymbol{A})$ and after $15 \mathrm{~min}$ of caCaMKI infusion $(\boldsymbol{B})$. Insets, Bottom traces are averages 政 $199 \mathrm{mEPSC}$ for $\boldsymbol{A}$ and $\boldsymbol{B}$, respectively; top traces are the corresponding variances. Calibrations are the same for $\boldsymbol{A}$ and conductance for cells in $\boldsymbol{C}$ with break in values normalized to 1. $E$, Changes in mEPSC amplitude as a function of channel conduc-

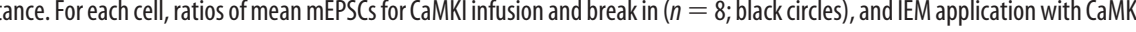
terminus of GluR1. CaMKII and CaMKI (activated by CaMKK) were assayed for ${ }^{32} \mathrm{P}$ incorporation into the C terminus of GluR1 (Barria et al., 1997) and the synthetic peptide substrate syntide-2. (PM, Counts per minute. Note the different scales for CaMKI and CaMKII and that heat-inactived CaMKI (used in Fig. 1) was catalytically inactive. ${ }^{*} p<0.05$; ${ }^{* *} p<0.01 ;{ }^{* * *} p<0.001$.

dependent GluR1 insertion (Korkotian and Segal, 2007), into spines and synapses (Matsuzaki et al., 2004; Korkotian and Segal, 2007). Second, actin polymerization significantly increases in CA1 hippocampal spines during TBS-LTP (Lin et al., 2005) and is involved in LTP consolidation (Messaoudi et al., 2007; Rex et al., 2007). Last, CaMKI can modulate spine actin dynamics directly thorough activation of Rac (Saneyoshi et al., 2008) or indirectly through activation of Ras-ERK signaling (Schmitt et al., 2004), which in turn regulates actin polymerization (Futter et al., 2005).

To assess the role of actin, we infused latrunculin $\mathrm{A}$, which specifically binds actin monomers and potently inhibits actin polymerization, with or without coinfusion of active CaMKI. Infusion of latrunculin A alone gave a small ( $\sim 15 \%)$ but significant suppression of mEPSC amplitude (Fig. $3 A$ ), consistent with a role for the actin cytoskeleton in constitutive recycling of a pool of synaptic AMPARs (Park et al., 2004; Schulz et al., 2004; J. Lu et al., 2007). There was no effect of latrunculin on mEPSC kinetics (Fig. $3 B$ ), indicating no involvement of CP-AMPARs as expected, because they are absence in synapses under basal conditions (Fig. 1). However, coinfusion of latrunculine A with CaMKI completely blocked the effects of CaMKI alone (dashed lines) on AMPAR mEPSC amplitude and decay kinetics (Fig. 3C,D). Because both the changes in amplitude and decay kinetics were strongly 

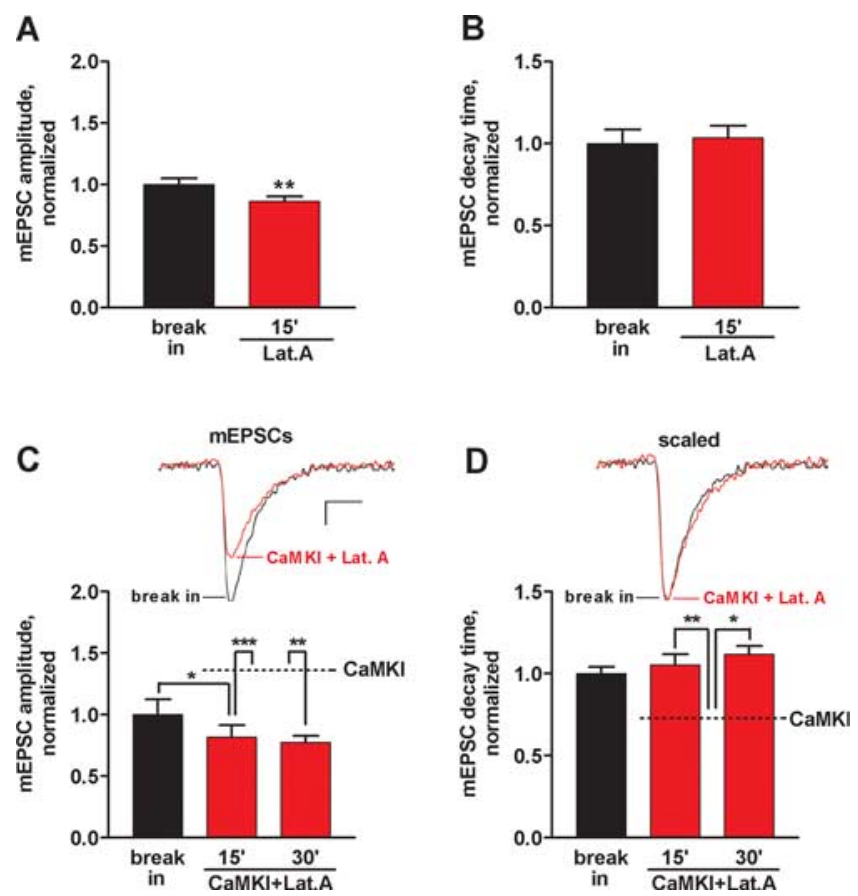

Figure 3. Actin polymerization is required for CaMKI-mediated synaptic expression of CPAMPARs. $\boldsymbol{A}, \boldsymbol{B}$, Under basal conditions, intracellular infusion of latrunculin $\mathrm{A}(2 \mu \mathrm{M}, n=7)$ to inhibit actin polymerization resulted in a small inhibition of $m E P S C$ amplitude $(\boldsymbol{A})$ but not decay kinetics $(\boldsymbol{B})$. C, D, Coinfusion of latrunculin A (Lat.A) with activated CaMKI blocked both the increases in mEPSC amplitude ( $\boldsymbol{C}, n=7$ for $15 \mathrm{~min}$ and $n=3$ for $30 \mathrm{~min}$ ) and acceleration in kinetics $(\boldsymbol{D})$ caused by infused CaMKI alone (effects of CaMKI alone are indicated by dashed lines and taken from Fig. 1). Insets, Averaged mEPSCs recorded during first $2 \mathrm{~min}$ (break in) and after 15 min of coinfusion (caCaMKI + latrunculin). Calibration: 1 pA, 5 ms. ${ }^{*} p<0.05$; ${ }^{* *} p<0.01$; ${ }^{* * *} p<0.001$. Error bars indicate SEM.

inhibited, this indicates an essential role of actin polymerization in CaMKI-mediated recruitment of synaptic CP-AMPARs.

Dendritic CP-AMPARs are excluded from the synapse basally Our experiments in Figure 1 demonstrate a lack of synaptic CPAMPARs under basal conditions, but they are recruited to the synapse within 15 min of active CaMKI infusion. Recruitment of AMPARs to synapses can occur from intracellular pools or via membrane diffusion from extrasynaptic sites (Cognet et al., 2006). Therefore, we wanted to know whether these CPAMPARs preexist extrasynaptically under basal conditions or whether they are assembled de novo by CaMKI stimulation and trafficked into the synapse. When the lysate from mature basal hippocampal neurons is immunodepleted of GluR2, there is still significant GluR1 remaining in the supernatant (Oh and Derkach, 2005), indicating a significant fraction of preexisting GluR2-lacking, GluR1-containing AMPARs (i.e., CP-AMPARs). To test whether these nonsynaptic (Fig. 1) CP-AMPARs might reside on the surface of dendrites, AMPA was ionophoretically applied. Consistent with this possibility, local dendritic application of AMPA under basal conditions revealed significant current rectification (Fig. 4A,B), a hallmark of CP-AMPARs (Oh and Derkach, 2005; Isaac et al., 2007). Furthermore, IEM suppressed this rectifying component of dendritic AMPA-currents, but did not block synaptic mEPSCs in the same cells (Fig. 4C,D). Unfortunately, we were unable to measure rectification of AMPA mEPSCs because increased basal noise at positive potentials and concerns over the long duration of depolarization (several minutes) required for averaging outward mEPSCs. Prolonged membrane depolarization can result in secretion of neurotrophic factors, such as BDNF, from hippocampal pyramidal neurons and activation of Ras (Iida et al., 2001; Kolarow et al., 2007). In turn, BDNF can induce trafficking to and insertion of GluR1 homomers at synapses of hippocampal neurons, and phosphorylation of Ser831 (Caldeira et al., 2007; Nakata and Nakamura, 2007). Thus, such manipulations could lead to potentially altered trafficking of synaptic AMPARs and aberrant rectification. Nevertheless, our observations indicate a significant reservoir of dendritic, extrasynaptic CP-AMPARs in hippocampal pyramidal neurons.

\section{Hippocampal CA1 LTP induced by TBS, but not HFS, transiently recruits synaptic CP-AMPARs via CaM-kinase kinase}

Having established a biochemical role for CaMKI in the recruitment of CP-AMPARs to synapses in cultured hippocampal neurons, we next asked whether this also occurs under more physiological circumstances such as hippocampal slice LTP. We have shown previously that inhibition of CaMKK, the upstream activator of CaMKI, partially suppresses TBS-LTP (Schmitt et al., 2005). Under our experimental conditions, $30 \mu \mathrm{M}$ IEM was selective for CP-AMPRs: it did not inhibit NMDARs (Fig. 5A) and gave no inhibition of basal synaptic transmission (Fig. $5 B$ ). This latter result is consistent with the absence of CP-AMPARs at naive Schaffer collateral-CA1 synapses in wild-type rodents whereas IEM gives almost complete inhibition (90\%) of basal transmission in the GluR2 knock-out mouse (Gray et al., 2007), consistent with the specificity of IEM for GluR2-lacking AMPARs. Importantly, IEM treatment immediately after TBS stimulation suppressed LTP by $\sim 40 \%$ (Fig. $5 C$ ), indicating that rapid synaptic incorporation of CP-AMPARs had occurred. Intriguingly, the reduction in TBS-LTP by IEM is similar in magnitude and onset to what we observed previously after inhibition of CaMKK by STO-609 (Schmitt et al., 2005). STO-609 potently inhibits CaMKK in vitro with little effect on numerous other protein kinases (Tokumitsu et al., 2002). Furthermore, STO-609 has no effect on basal synaptic transmission, paired-pulse facilitation, or CaMKII activation during LTP, yet it prevents activation of CaMKI by CaMKK during TBS-LTP (Schmitt et al., 2005). As expected, STO-609 pretreatment suppressed TBS-LTP identical to IEM-treatment (Fig. 5C). Importantly, in STO-treated slices IEM had no additional effect on LTP, indicating that synaptic incorporation of CP-AMPARs requires CaMKK signaling.

It was reported previously that incorporation of CP-AMPARs after pairing-induced LTP is transient and lasts $<30 \mathrm{~min}$ (Plant et al., 2006). Likewise, IEM had no effect when applied $20 \mathrm{~min}$ after TBS-LTP (Fig. 5D), indicative of a very transient expression of CP-AMPARs in the early phase of TBS-LTP. In contrast, HFSLTP has been reported to occur independently of CP-AMPARs (Adesnik and Nicoll, 2007; Gray et al., 2007), although this finding appears to be age-dependent (Y. Lu et al., 2007), so we also investigated this protocol. Indeed, we also did not observe any effect of IEM on HFS-LTP (Fig. 5E), indicating that CP-AMPARs were not recruited to synapses. Our TBS- and HFS-LTP experiments were similar except for the pattern of activity: both used three one second long trains of either TBS (4 pulses/burst at 100 $\mathrm{Hz}, 5$ bursts at $5 \mathrm{~Hz})$ or HFS $(100 \mathrm{~Hz}, 1 \mathrm{~s})$ spaced $20 \mathrm{~s}$ apart. If CaMKK and CaMKI regulate LTP specifically through the recruitment of CP-AMPARs, one would predict that CaMKK inhibition by STO-609 would have no effect on HFS-LTP, which did not use CP-AMPARs, and this is exactly what we observed (Fig. $5 E$ ). Thus, the pattern of activity used to generate LTP appears to 
be critical for the recruitment of synaptic CP-AMPARs via CaMKK/CaMKI.

\section{Quantification of synaptic potentiation by subunit recomposition}

How efficient is subunit recomposition for synaptic potentiation? We estimated the relative number of CP-AMPARs, which exhibit high conductance compared with (edited)GluR2-containing AMPARs residing at the synapse basally, needed to account for both potentiation and rectification of AMPARs at SC-CA1 synapses (see Appendix). CP-AMPARs are likely to be predominantly GluR1 homomers because expression levels of GluR3 and GluR4 in the mature hippocampus are very low compared with GluR1 and GluR2 (Monyer et al., 1991; Craig et al., 1993; Geiger et al., 1995; Wenthold et al., 1996; Sans et al., 2003). We calculated the fraction of CP-AMPARs added to the existing synaptic population of $\mathrm{Ca}^{2+}$ impermeable AMPARs to account for changes in both rectification properties of synaptic AMPARs and synaptic strength. Based on rectification measurements (Appendix, Equation 5), the fraction is $\leq 0.015$. Thus, the addition of only $2 \%$ of GluR1 homomers can produce the rectification seen at CA1 synapses during pairing-induced early LTP (E-LTP) (Plant et al., 2006).

The fraction of newly inserted GluR1 homomers can also be calculated using an alternative approach based on the increase in synaptic strength at negative membrane potentials (Appendix, Equation 8). For pairing-induced LTP (Plant et al., 2006), this ratio is $\leq 4.3 \%$. Furthermore, our observations independently suggests that for both TBS-LTP and potentiation by CaMKI infusion, the ratio is $1.6-2.1 \%$, considering $\sim 30-40 \%$ increases in synaptic strength attributable to CP-AMPARs (Figs. 1, 5). Importantly, these calculations provide relative changes in receptor number and are therefore independent of their initial number at synapses. This is noteworthy because excitatory synapses are heterogeneous with respect to AMPAR number (Nusser et al., 1998; Matsuzaki et al., 2004). Thus, both approaches substantiate each other and indicate that a small fraction $(<5 \%)$ of newly recruited GluR1 homomers can produce the observed changes in both rectification and synaptic strength for the three different stimulation paradigms (pairing-induced LTP, TBS-LTP, and active CaMKI infusion) (Fig. 6). Such an efficient mechanism for enhancing postsynaptic strength via trafficking of GluR1 homomers may be critical for rapid postsynaptic potentiation before subsequent slower structural remodeling and enlargement of synaptic spines (Matsuzaki et al., 2004; Nagerl et al., 2004; Kopec et al., 2007; Korkotian and Segal, 2007).

\section{Discussion}

Our principal finding is that the CaMKK/CaMKI signaling pathway can recruit CP-AMPARs to synapses (Figs. 1, 2, 5). Based on the unique properties of CP-AMPARs (Isaac et al., 2007), we used several criteria to detect their presence in synapses. Thus, inhibition by IEM 1460 of potentiated mEPSCs, acceleration in the decay kinetics, changes in channel conductance of synaptic AM-
PARs, and lack of GluR1 phosphorylation by CaMKI all point to the recruitment of synaptic CP-AMPARs mediated by CaMKK/ CaMKI. Although CaMKII can phosphorylate Ser831 of GluR1 in GluR2-containing AMPARs that exist at synapses under basal conditions, this does not result in increased channel conductance as occurs with homomeric GluR1 (Derkach et al., 1999; Derkach, 2003). Thus, it requires CaMKI-mediated recruitment of synaptic GluR2-lacking AMPARs, which by themselves have higher conductance, for the CaMKII phosphorylation to further increase channel conductance. Thus, these two CaM-kinases act in concert to achieve synaptic potentiation through recruitment and phosphorylation of CP-AMPARs (Fig. 6).

It is likely that CaMKK/CaMKI-regulated actin dynamics (Saneyoshi et al., 2008) are necessary for $\mathrm{Ca}^{2+}$-dependent trafficking of GluR1 homomers (Korkotian and Segal, 2007) from a dendritic, extrasynaptic pool to synapses (Figs. 3, 4). This would be consistent with the observation that actin polymerization significantly increases in CA1 hippocampal spines during TBS-LTP (Lin et al., 2005). Thus, TBS-LTP, which is likely more physiological than HFS- or pairing-induced LTP, promoted rapid but transient synaptic incorporation of CP-AMPARs that required CaMKK signaling (Fig. 5). Importantly, our LTP experiments used native AMPARs and endogenous signaling pathways in intact neurons. Although studies of transfected GluR1 in neurons can allow unique analyses of receptor trafficking, it is likely this manipulation promotes nonphysiological levels of GluR1 that could artificially promote formation of GluR1 homomers.

Our observations also demonstrate that recruitment of native CP-AMPARs depends on the pattern of synaptic activity used to induce LTP because TBS, but not HFS (Adesnik and Nicoll, 2007; Gray et al., 2007), resulted in transient expression of CPAMPARs (Fig. 5). It is well known that various LTP induction protocols can trigger different signaling pathways including 

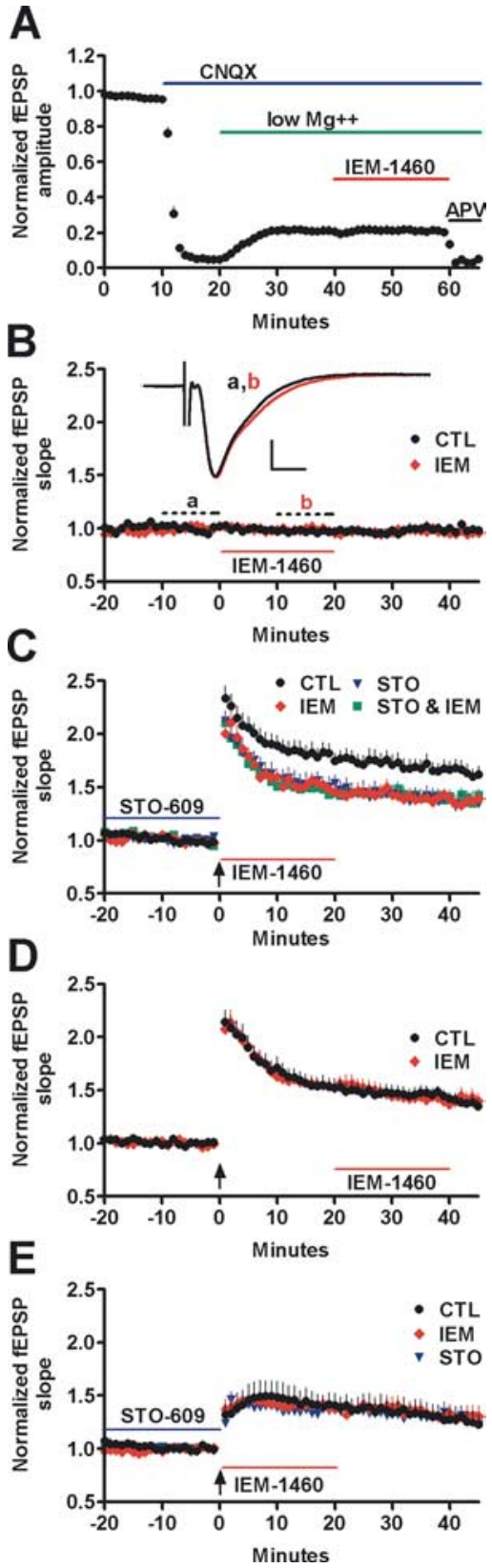

Figure 5. CaMKK regulates transient synaptic CP-AMPAR expression during TBS-LTP. Field recordings (fEPSPS) from area CA1 (stratum radiatum) elicited by SC-stimulation (see Materials and Methods) in acute hippocampal slices (4- to 6-week-old rat). $\boldsymbol{A}$ confirms that IEM (30 $\mu \mathrm{m}$ ) does not inhibit NMDARs (pharmacologically isolated with $10 \mu \mathrm{m}$ (NQX in $0.1 \mathrm{~mm} \mathrm{Mg}^{2+}$ ), and $\boldsymbol{B}$ demonstrates that IEM $(30 \mu \mathrm{m})$ has no effect on basal synaptic transmission in naive slices $(n=6)$. Inset, Representative fEPSP averages before (a, black trace) and during ( $b$, red trace). Calibration: $0.5 \mathrm{mV}, 5 \mathrm{~ms}$. C, Application of IEM immediately after TBS (Materials and Methods) suppressed LTP 40\% compared with control (CTL). This IEM-sensitive component of LTP was absent in slices pretreated with the CaMKK inhibitor ST0-609 $(5 \mu \mathrm{M})$, demonstrating rapid recruitment of synaptic (P-AMPARs downstream of CaMKK ( $n=9-10)$. D, Application of IEM 20 min after TBS did not alter LTP expression, indicating that synaptic incorporation of CP-AMPARs is transient $(n=9)$. $\boldsymbol{E}$, In contrast to TBS-LTP, HFS-LTP (see Materials and Methods) was not affected by ST0-609 pretreatment or treatment with IEM immediately after HFS $(n=10)$.

CaMKI, CaMKII, PKA (protein kinase A), PKC, and ERK (Selcher et al., 2003; Yasuda et al., 2003; Schmitt et al., 2005). Even the same protocol can use different pathways depending on the developmental age (Yasuda et al., 2003; Y. Lu et al., 2007). It appears that multiple signaling pathways may mediate TBS-LTP because inhibition of CaMKK only gives a $40 \%$ suppression.
Why do synapses use CP-AMPARs to initiate plasticity? Insertion of highly conductive synaptic CP-AMPARs from a dendritic extrasynaptic pool, would allow postsynaptic potentiation via AMPAR trafficking to occur rapidly. This higher conductance of CP-AMPARs compared with GluR2(edited)-containing AMPARs, and their ability to be further potentiated by phosphorylation of GluR1 at Ser831 (Oh and Derkach, 2005), make them ideally suited to initiate LTP where both rapid trafficking and increased conductance of postsynaptic AMPARs are established mechanisms (Benke et al., 1998; Luthi et al., 2004). Based on these considerations, we calculated that $<5 \%$ of existing synaptic GluR1/GluR2 receptors would have to be augmented by homomeric GluR1 (Fig. 6) to account for the degree of potentiation observed in three different forms of synaptic potentiation by CPAMPARs: pairing-induced LTP (Plant et al., 2006), TBS-LTP (Fig. 5), and CaMKI infusion (Fig. 1). This mechanism for initial LTP expression appears to be the inverse of what happens during LTD at young ( $<3$ weeks) cerebellar stellate synapses and mossy fiber-CA3 pyramidal cell synapses. These synapses contain CPAMPARs that are replaced by GluR2-containing AMPARs (Ho et al., 2007) from an extrasynaptic pool (Liu and Cull-Candy, 2000, 2005). This subunit recomposition appears to involve interaction of the GluR2 subunit with the proteins PICK1 (PKC-interacting protein 1) (Ho et al., 2007) and GRIP (glutamate receptorinteracting protein) (Liu and Cull-Candy, 2005).

Is rapid regulation of synaptic strength the only function of CP-AMPARs, and why does their expression in CA1-LTP last $<20-25$ min (Fig. 5D) (Plant et al., 2006)? Because these receptors have high $\mathrm{Ca}^{2+}$ and $\mathrm{Zn}^{2+}$ permeability at resting membrane potentials, their prolonged synaptic activation could lead to cell death (Calderone et al., 2004). Indeed, several neuropathologies associated with cell death are attributed to the expression of CPAMPARs (for review, see Kwak and Weiss, 2006; Liu and Zukin, 2007). It is also possible that either the transient $\mathrm{Ca}^{2+}$ influx through CP-AMPARs or the synaptic insertion of the GluR1 C terminus (Kopec et al., 2007) regulate unique signaling events important for subsequent stable LTP expression and structural plasticity of spines. One attractive candidate that we are investigating is modulation of structural plasticity resulting from actin and CaMKs, which mediate changes in spine morphology (Matsuzaki et al., 2004; Nagerl et al., 2004; Saneyoshi et al., 2008).

\section{Appendix}

\section{Quantifying the fraction of CP-AMPARs for} synaptic potentiation

We estimated how many CP-AMPARs would need to be inserted into a CA1 synapse to account for the observed synaptic potentiation. Our calculations are based on the conclusion that these CP-AMPARs are predominantly GluR1 homomers because expression levels of GluR3 and GluR4 in the mature hippocampus are very low compared with GluR1 and GluR2 (Monyer et al., 1991; Craig et al., 1993; Geiger et al., 1995; Wenthold et al., 1996; Sans et al., 2003).

Subunit recomposition is accompanied by increased rectification of AMPAR-mediated synaptic currents (Plant et al., 2006), and one can calculate the rectification index, RI, as follows:

$$
\mathrm{RI}=\mathrm{EPSC}_{\mathrm{LTP}}(-60 \mathrm{mV}) / \mathrm{EPSC}_{\mathrm{LTP}}(40 \mathrm{mV}),
$$

where $\operatorname{EPSC}_{\mathrm{LTP}}(-60 \mathrm{mV})$ and $\mathrm{EPSC}_{\mathrm{LTP}}(40 \mathrm{mV})$ are amplitudes of EPSCs at -60 and $40 \mathrm{mV}$ of membrane potential, respectively, during E-LTP. One can write for the enhanced EPSC at $-60 \mathrm{mV}$ as follows: 


$$
\begin{array}{r}
\mathrm{EPSC}_{\mathrm{LTP}}(-60 \mathrm{mV})=\left(\gamma_{\mathrm{CI}} P_{o, \mathrm{CI}} N_{\mathrm{CI}}+\right. \\
\left.k \gamma_{\mathrm{CP}} P_{o, \mathrm{CP}} N_{\mathrm{CP}}\right) E(-60),
\end{array}
$$

where $\gamma_{\mathrm{CI}}$ and $\gamma_{\mathrm{CP}}$ are channel conductances, $P_{o, \mathrm{CI}}$ and $P_{o, \mathrm{CP}}$ are open probabilities and $N_{\mathrm{CI}}$ and $N_{\mathrm{CP}}$ are the number of GluR2-containing heteromers (designated CI for calcium impermeable) and GluR1 homomers (designated CP for calcium permeable) in a synapse, respectively. $E(-60)$ is the electrochemical potential for GluR2-containing AMPARs, and $k$ is a coefficient accounting for the difference in electrochemical potentials between GluR2-containing heteromers and GluR1 homomers. However, in contrast to -60 $\mathrm{mV}$, the contribution of GluR 1 homomers to postsynaptic current at $40 \mathrm{mV}$ is negligible because it is reduced by a factor of 45 (Derkach et al., 1999; Oh and Derkach, 2005) as these channels are blocked at this membrane potential by intracellular polyamines (Donevan and Rogawski, 1995; Bowie et al., 1998), producing rectification of the postsynaptic current during E-LTP (Plant et al., 2006). This is translated into

$$
\mathrm{EPSC}_{\mathrm{LTP}}(40 \mathrm{mV})=\gamma_{\mathrm{CI}} P_{o, \mathrm{CI}} N_{\mathrm{cI}} E(40),
$$

where $E(40)$ is an electrochemical potential for GluR1 homomers. Substituting Equations 2 and 3 into 1 yields the following:

$$
\mathrm{RI}=\left[1+\left(k \gamma_{\mathrm{CP}} P_{o, \mathrm{CP}} N_{\mathrm{CP}}\right) /\left(\gamma_{\mathrm{CI}} P_{o, \mathrm{CI}} N_{\mathrm{CI}}\right)\right] E(-60) / E(40) .
$$

In this equation, the ratio $N_{\mathrm{CP}} / N_{\mathrm{CI}}$ represents the fraction of new GluR1 homomers incorporated into a potentiated synapse. Solving for this ratio gives the following:

$$
N_{\mathrm{CB}} / N_{\mathrm{CI}}=[\mathrm{RI} E(40) / E(-60)-1] \gamma_{\mathrm{CI}} P_{o, \mathrm{CI}} /\left(k \gamma_{\mathrm{CP}} P_{o, \mathrm{CP}}\right) .
$$

There are, however, two different types of CA1 synapses: those that showed an increase in single-channel conductance after LTP induction and those that did not (Benke et al., 1998; Lüthi et al., 2004). Importantly, these two types of synapses were also different in their basal channel conductances. Those synapses showing an increase in $\gamma$ had baseline $\gamma$ values of $4.8 \mathrm{pS}$ versus $7.2 \mathrm{pS}$ in those synapses without changes in $\gamma$ (Benke et al., 1998). We therefore used the value $4.8 \mathrm{pS}$ for nonpotentiated synapses in our modeling (see also comments of low $\gamma$ estimates in Materials and Methods). AMPARs are phosphorylated at Ser831 in GluR1 during LTP (Barria et al., 1997b; Lee et al., 2000), and channel conductance of phosphorylated GluR1 has been measured [taken as $18.9 \mathrm{pS}$ from the studies by Derkach et al. (1999) and Oh and Derkach (2005)]. GluR2 subunit also controls open probability of AMPARs, and we found that GluR1/GluR2 heteromers have at least a fourfold lower open probability compared with GluR1 homomers (Oh and Derkach, 2005), so we can write $P_{o, \mathrm{CI}} / P_{o, \mathrm{CP}}$ as $\leq 0.25$. The value of $k$ was given as 1.15 , assuming that currents through GluR2-containing AMPARs and GluR1 homomers reverse at 0 and $9 \mathrm{mV}$, respectively. Substitution of all of these values and RI [taken as 1.85 from the study by Plant et al. (2006)] in Equation 5 yields $N_{\mathrm{CP}} / N_{\mathrm{CI}} \leq 0.015$. Thus, insertion of $\sim 2 \%$ of GluR1 homomers is sufficient to produce the rectification seen at CA1 synapses during E-LTP.

The number of newly inserted GluR1 homomers can be also calculated in an alternative way based on the increase in synaptic strength at negative membrane potentials. Thus,

$$
\mathrm{EPSC}_{\mathrm{LTP}}(-60 \mathrm{mV}) / \mathrm{EPSC}_{\text {base }}(-60 \mathrm{mV})=1+\Delta \mathrm{EPSC},
$$

where $\mathrm{EPSC}_{\text {basal }}(-60 \mathrm{mV})$ is a baseline EPSC before LTP induction and can be expressed as follows:

$$
\operatorname{EPSC}_{\text {basal }}(-60 \mathrm{mV})=\gamma_{\mathrm{CI}} P_{o, \mathrm{CI}} N_{\mathrm{CI}} E(-60),
$$

where $\triangle \mathrm{EPSC}$ is an increase in synaptic strength during E-LTP. After substituting Equations 2 and 7 into 6 and solving for $N_{\mathrm{CP}} /$ $N_{\mathrm{CI}}$, one can write

$$
N_{\mathrm{CP}} / N_{\mathrm{CI}}=\Delta \operatorname{EPSC}\left(\gamma_{\mathrm{CI}} P_{o, \mathrm{CI}} / k \gamma_{\mathrm{CP}} P_{o, \mathrm{CP}}\right) .
$$

At $80 \%$ of potentiation during pairing-induced E-LTP (Plant et al., 2006), this ratio is $4.3 \%$. Furthermore, for both TBS-LTP and potentiation by CaMKI infusion, the ratio is $1.6-2.1 \%$, assuming $\sim 30-40 \%$ increases in synaptic strength associated with CP-AMPARs (Figs. 1, 5). It is noteworth that Equations 5 and 8 are general expressions estimating relative changes in the number of receptors and, thus, are independent of the initial number of AMPARs at a synapse.

\section{References}

Adesnik H, Nicoll RA (2007) Conservation of glutamate receptor 2-containing AMPA receptors during long-term potentiation. J Neurosci 27:4598-4602.

Barria A, Derkach V, Soderling T (1997a) Identification of the $\mathrm{Ca}^{2+}$ / calmodulin-dependent protein kinase II regulatory phosphorylation site in the alpha-amino-3-hydroxyl-5-methyl-4-isoxazole-propionate-type glutamate receptor. J Biol Chem 272:32727-32730.

Barria A, Muller D, Derkach V, Griffith LC, Soderling TR (1997b) Regulatory phosphorylation of AMPA-type glutamate receptors by CaM-KII during long-term potentiation. Science 276:2042-2045.

Benke TA, Luthi A, Isaac JT, Collingridge GL (1998) Modulation of AMPA receptor unitary conductance by synaptic activity. Nature 393:793-797.

Benke TA, Luthi A, Palmer MJ, Wikstrom MA, Anderson WW, Isaac JT, Collingridge GL (2001) Mathematical modelling of non-stationary fluc- 
tuation analysis for studying channel properties of synaptic AMPA receptors. J Physiol 537:407-420.

Bergles DE, Diamond JS, Jahr CE (1999) Clearance of glutamate inside the synapse and beyond. Curr Opin Neurobiol 9:293-298.

Bowie D, Lange GD, Mayer ML (1998) Activity-dependent modulation of glutamate receptors by polyamines. J Neurosci 18:8175-8185.

Buldakova SL, Vorobjev VS, Sharonova IN, Samoilova MV, Magazanik LG (1999) Characterization of AMPA receptor populations in rat brain cells by the use of subunit-specific open channel blocking drug, IEM-1460. Brain Res 846:52-58.

Buldakova SL, Kim KK, Tikhonov DB, Magazanik LG (2007) Selective blockade of $\mathrm{Ca}^{2+}$ permeable AMPA receptors in CA1 area of rat hippocampus. Neuroscience 144:88-99.

Caldeira MV, Melo CV, Pereira DB, Carvalho R, Correia SS, Backos DS, Carvalho AL, Esteban JA, Duarte CB (2007) Brain-derived neurotrophic factor regulates the expression and synaptic delivery of alpha-amino3-hydroxy-5-methyl-4-isoxazole propionic acid receptor subunits in hippocampal neurons. J Biol Chem 282:12619-12628.

Calderone A, Jover T, Mashiko T, Noh KM, Tanaka H, Bennett MV, Zukin RS (2004) Late calcium EDTA rescues hippocampal CA1 neurons from global ischemia-induced death. J Neurosci 24:9903-9913.

Cognet L, Groc L, Lounis B, Choquet D (2006) Multiple routes for glutamate receptor trafficking: surface diffusion and membrane traffic cooperate to bring receptors to synapses. Sci STKE 2006:pe13.

Craig AM, Blackstone CD, Huganir RL, Banker G (1993) The distribution of glutamate receptors in cultured rat hippocampal neurons: postsynaptic clustering of AMPA-selective subunits. Neuron 10:1055-1068.

Derkach V, Barria A, Soderling TR (1999) $\mathrm{Ca}^{2+} /$ calmodulin-kinase II enhances channel conductance of alpha-amino-3-hydroxy-5-methyl-4isoxazolepropionate type glutamate receptors. Proc Natl Acad Sci USA 96:3269-3274.

Derkach VA (2003) Silence analysis of AMPA receptor mutated at the CaMkinase II phosphorylation site. Biophys J 84:1701-1708.

Derkach VA, Oh MC, Guire ES, Soderling TR (2007) Regulatory mechanisms of AMPA receptors in synaptic plasticity. Nat Rev Neurosci 8:101-113.

Donevan SD, Rogawski MA (1995) Intracellular polyamines mediate inward rectification of $\mathrm{Ca}^{2+}$-permeable alpha-amino-3-hydroxy-5methyl-4-isoxazolepropionic acid receptors. Proc Natl Acad Sci USA 92:9298-9302.

Feder R, Ranck Jr JB (1973) Studies on single neurons in dorsal hippocampal formation and septum in unrestrained rats. II. Hippocampal slow waves and theta cell firing during bar pressing and other behaviors. Exp Neurol 41:532-555.

Futter M, Uematsu K, Bullock SA, Kim Y, Hemmings Jr HC, Nishi A, Greengard P, Nairn AC (2005) Phosphorylation of spinophilin by ERK and cyclin-dependent PK 5 (Cdk5). Proc Natl Acad Sci USA 102:3489-3494.

Gebhardt C, Cull-Candy SG (2006) Influence of agonist concentration on AMPA and kainate channels in CA1 pyramidal cells in rat hippocampal slices. J Physiol (Lond) 573:371-394.

Geiger JR, Melcher T, Koh DS, Sakmann B, Seeburg PH, Jonas P, Monyer H (1995) Relative abundance of subunit mRNAs determines gating and $\mathrm{Ca}^{2+}$ permeability of AMPA receptors in principal neurons and interneurons in rat CNS. Neuron 15:193-204.

Gray EE, Fink AE, Sarinana J, Vissel B, O’Dell TJ (2007) Long-term potentiation in the hippocampal CA1 region does not require insertion and activation of GluR2-lacking AMPA receptors. J Neurophysiol 98:2488-2492.

Grosskreutz J, Zoerner A, Schlesinger F, Krampfl K, Dengler R, Bufler J (2003) Kinetic properties of human AMPA-type glutamate receptors expressed in HEK293 cells. Eur J Neurosci 17:1173-1178.

Ho MT, Pelkey KA, Topolnik L, Petralia RS, Takamiya K, Xia J, Huganir RL, Lacaille JC, McBain CJ (2007) Developmental expression of $\mathrm{Ca}^{2+}$ permeable AMPA receptors underlies depolarization-induced long-term depression at mossy fiber CA3 pyramid synapses. J Neurosci 27:11651-11662.

Iida N, Namikawa K, Kiyama H, Ueno H, Nakamura S, Hattori S (2001) Requirement of Ras for the activation of mitogen-activated protein kinase by calcium influx, cAMP, and neurotrophin in hippocampal neurons. J Neurosci 21:6459-6466.

Isaac JT, Ashby M, McBain CJ (2007) The role of the GluR2 subunit in AMPA receptor function and synaptic plasticity. Neuron 54:859-871.
Ju W, Morishita W, Tsui J, Gaietta G, Deerinck TJ, Adams SR, Garner CC, Tsien RY, Ellisman MH, Malenka RC (2004) Activity-dependent regulation of dendritic synthesis and trafficking of AMPA receptors. Nat Neurosci 7:244-253.

Kolarow R, Brigadski T, Lessmann V (2007) Postsynaptic secretion of BDNF and NT-3 from hippocampal neurons depends on calcium calmodulin kinase II signaling and proceeds via delayed fusion pore opening. J Neurosci 27:10350-10364.

Kopec CD, Real E, Kessels HW, Malinow R (2007) GluR1 links structural and functional plasticity at excitatory synapses. J Neurosci 27:13706-13718

Korkotian E, Segal M (2007) Morphological constraints on calcium dependent glutamate receptor trafficking into individual dendritic spine. Cell Calcium 42:41-57.

Kwak S, Weiss JH (2006) Calcium-permeable AMPA channels in neurodegenerative disease and ischemia. Curr Opin Neurobiol 16:281-287.

Laezza F, Dingledine R (2004) Voltage-controlled plasticity at GluR2deficient synapses onto hippocampal interneurons. J Neurophysiol 92:3575-3581.

Lee HK, Barbarosie M, Kameyama K, Bear MF, Huganir RL (2000) Regulation of distinct AMPA receptor phosphorylation sites during bidirectional synaptic plasticity. Nature 405:955-959.

Li S, Tian X, Hartley DM, Feig LA (2006) Distinct roles for Ras-guanine nucleotide-releasing factor 1 (Ras-GRF1) and Ras-GRF2 in the induction of long-term potentiation and long-term depression. J Neurosci 26:1721-1729.

Lin B, Kramar EA, Bi X, Brucher FA, Gall CM, Lynch G (2005) Theta stimulation polymerizes actin in dendritic spines of hippocampus. J Neurosci 25:2062-2069.

Liu SJ, Cull-Candy SG (2005) Subunit interaction with PICK and GRIP controls $\mathrm{Ca}^{2+}$ permeability of AMPARs at cerebellar synapses. Nat Neurosci 8:768-775.

Liu SJ, Zukin RS (2007) $\mathrm{Ca}^{2+}$-permeable AMPA receptors in synaptic plasticity and neuronal death. Trends Neurosci 30:126-134.

Liu SQ, Cull-Candy SG (2000) Synaptic activity at calcium-permeable AMPA receptors induces a switch in receptor subtype. Nature 405:454-458.

Lu J, Helton TD, Blanpied TA, Racz B, Newpher TM, Weinberg RJ, Ehlers MD (2007) Postsynaptic positioning of endocytic zones and AMPA receptor cycling by physical coupling of dynamin-3 to Homer. Neuron 55:874-889.

Lu Y, Allen M, Halt AR, Weisenhaus M, Dallapiazza RF, Hall DD, Usachev YM, McKnight GS, Hell JW (2007) Age-dependent requirement of AKAP150-anchored PKA and GluR2-lacking AMPA receptors in LTP. EMBO J 26:4879-4890.

Lüthi A, Wikstrom MA, Palmer MJ, Matthews P, Benke TA, Isaac JT, Collingridge GL (2004) Bi-directional modulation of AMPA receptor unitary conductance by synaptic activity. BMC Neurosci 5:44.

Magazanik LG, Buldakova SL, Samoilova MV, Gmiro VE, Mellor IR, Usherwood PN (1997) Block of open channels of recombinant AMPA receptors and native AMPA/kainate receptors by adamantane derivatives. J Physiol (Lond) 505:655-663.

Mainen ZF, Malinow R, Svoboda K (1999) Synaptic calcium transients in single spines indicate that NMDA receptors are not saturated. Nature 399:151-155.

Mameli M, Balland B, Lujan R, Luscher C (2007) Rapid synthesis and synaptic insertion of GluR2 for mGluR-LTD in the ventral tegmental area. Science 317:530-533.

Matsuzaki M, Honkura N, Ellis-Davies GC, Kasai H (2004) Structural basis of long-term potentiation in single dendritic spines. Nature 429:761-766.

Messaoudi E, Kanhema T, Soule J, Tiron A, Dagyte G, da Silva B, Bramham CR (2007) Sustained Arc/Arg3.1 synthesis controls long-term potentiation consolidation through regulation of local actin polymerization in the dentate gyrus in vivo. J Neurosci 27:10445-10455.

Monyer H, Seeburg PH, Wisden W (1991) Glutamate-operated channels: developmentally early and mature forms arise by alternative splicing. Neuron 6:799-810.

Nagerl UV, Eberhorn N, Cambridge SB, Bonhoeffer T (2004) Bidirectional activity-dependent morphological plasticity in hippocampal neurons. Neuron 44:759-767.

Nakata H, Nakamura S (2007) Brain-derived neurotrophic factor regulates 
AMPA receptor trafficking to post-synaptic densities via IP3R and TRPC calcium signaling. FEBS Lett 581:2047-2054.

Nusser Z, Lujan R, Laube G, Roberts JD, Molnar E, Somogyi P (1998) Cell type and pathway dependence of synaptic AMPA receptor number and variability in the hippocampus. Neuron 21:545-559.

Oh MC, Derkach VA (2005) Dominant role of the GluR2 subunit in regulation of AMPA receptors by CaMKII. Nat Neurosci 8:853-854.

Otto T, Eichenbaum H, Wiener SI, Wible CG (1991) Learning-related patterns of CA1 spike trains parallel stimulation parameters optimal for inducing hippocampal long-term potentiation. Hippocampus 1:181-192.

Park M, Penick EC, Edwards JG, Kauer JA, Ehlers MD (2004) Recycling endosomes supply AMPA receptors for LTP. Science 305:1972-1975.

Plant K, Pelkey KA, Bortolotto ZA, Morita D, Terashima A, McBain CJ, Collingridge GL, Isaac JT (2006) Transient incorporation of native GluR2-lacking AMPA receptors during hippocampal long-term potentiation. Nat Neurosci 9:602-604.

Racca C, Catania MV, Monyer H, Sakmann B (1996) Expression of AMPAglutamate receptor B subunit in rat hippocampal GABAergic neurons. Eur J Neurosci 8:1580-1590.

Rex CS, Lin CY, Kramar EA, Chen LY, Gall CM, Lynch G (2007) Brainderived neurotrophic factor promotes long-term potentiation-related cytoskeletal changes in adult hippocampus. J Neurosci 27:3017-3029.

Samoilova MV, Buldakova SL, Vorobjev VS, Sharonova IN, Magazanik LG (1999) The open channel blocking drug, IEM-1460, reveals functionally distinct alpha-amino-3-hydroxy-5-methyl-4-isoxazolepropionate receptors in rat brain neurons. Neuroscience 94:261-268.

Saneyoshi T, Wayman G, Fortin D, Davare M, Hoshi N, Nozaki N, Natsume T, Soderling TR (2008) Activity-dependent synaptogenesis: regulation by a CaM-kinase kinase/CaM-kinase I/betaPIX signaling complex. Neuron 57:94-107.

Sans N, Vissel B, Petralia RS, Wang YX, Chang K, Royle GA, Wang CY, O'Gorman S, Heinemann SF, Wenthold RJ (2003) Aberrant formation of glutamate receptor complexes in hippocampal neurons of mice lacking the GluR2 AMPA receptor subunit. J Neurosci 23:9367-9373.

Schmitt JM, Wayman GA, Nozaki N, Soderling TR (2004) Calcium activation of ERK mediated by calmodulin kinase I. J Biol Chem 279:24064-24072.

Schmitt JM, Guire ES, Saneyoshi T, Soderling TR (2005) Calmodulindependent kinase kinase/calmodulin kinase I activity gates extracellularregulated kinase-dependent long-term potentiation. J Neurosci 25:1281-1290.
Schulz TW, Nakagawa T, Licznerski P, Pawlak V, Kolleker A, Rozov A, Kim J, Dittgen T, Kohr G, Sheng M, Seeburg PH, Osten P (2004) Actin/alphaactinin-dependent transport of AMPA receptors in dendritic spines: role of the PDZ-LIM protein RIL. J Neurosci 24:8584-8594.

Selcher JC, Weeber EJ, Christian J, Nekrasova T, Landreth GE, Sweatt JD (2003) A role for ERK MAP kinase in physiologic temporal integration in hippocampal area CA1. Learn Mem 10:26-39.

Sigworth FJ (1980) The variance of sodium current fluctuations at the node of Ranvier. J Physiol (Lond) 307:97-129.

Sutton MA, Ito HT, Cressy P, Kempf C, Woo JC, Schuman EM (2006) Miniature neurotransmission stabilizes synaptic function via tonic suppression of local dendritic protein synthesis. Cell 125:785-799.

Swanson GT, Kamboj SK, Cull-Candy SG (1997) Single-channel properties of recombinant AMPA receptors depend on RNA editing, splice variation, and subunit composition. J Neurosci 17:58-69.

Tokumitsu H, Enslen H, Soderling TR (1995) Characterization of a $\mathrm{Ca}^{2+}$ calmodulin-dependent protein kinase cascade. Molecular cloning and expression of calcium/calmodulin-dependent protein kinase kinase. J Biol Chem 270:19320-19324.

Tokumitsu H, Inuzuka H, Ishikawa Y, Ikeda M, Saji I, Kobayashi R (2002) STO-609, a specific inhibitor of the $\mathrm{Ca}^{2+} /$ calmodulin-dependent protein kinase kinase. J Biol Chem 277:15813-15818.

Traynelis SF, Silver RA, Cull-Candy SG (1993) Estimated conductance of glutamate receptor channels activated during EPSCs at the cerebellar mossy fiber-granule cell synapse. Neuron 11:279-289.

Wayman GA, Kaech S, Grant WF, Davare M, Impey S, Tokumitsu H, Nozaki N, Banker G, Soderling TR (2004) Regulation of axonal extension and growth cone motility by calmodulin-dependent protein kinase I. J Neurosci 24:3786-3794.

Wayman GA, Impey S, Marks D, Saneyoshi T, Grant WF, Derkach V, Soderling TR (2006) Activity-dependent dendritic arborization mediated by CaM-kinase I activation and enhanced CREB-dependent transcription of Wnt-2. Neuron 50:897-909.

Wenthold RJ, Petralia RS, Blahos II J, Niedzielski AS (1996) Evidence for multiple AMPA receptor complexes in hippocampal CA1/CA2 neurons. J Neurosci 16:1982-1989.

Yasuda H, Barth AL, Stellwagen D, Malenka RC (2003) A developmental switch in the signaling cascades for LTP induction. Nat Neurosci 6:15-16.

Zhu JJ, Qin Y, Zhao M, Van Aelst L, Malinow R (2002) Ras and Rap control AMPA receptor trafficking during synaptic plasticity. Cell 110:443-455. 